\title{
Determining Combining Ability in Sunflower (Helianthus annus L.)
}

\author{
U.K. Singh, Dhiraj Kumar and Rajesh Kumar* \\ Department of Plant Breeding and Genetics, Faculty of Agriculture, Dr. Rajendra Prasad \\ Central Agricultural University, Pusa (Samastipur) - 848125, Bihar, India \\ *Corresponding author
}

\section{A B S T R A C T}

\section{Keywords}

Sunflower, Hybrid, gca, sca, Line and Testers

\section{Article Info}

Accepted:

18 April 2018

Available Online:

10 May 2018
In the present investigation, all together 32 hybrids were made from crosses of four cytoplasmic male sterile lines and eight diverse testers in a line $\mathrm{x}$ tester matting design. These hybrids were evaluated during Rabi 2014-15 at TCA, Dholi Farm. The mean sum of squares for parents and crosses, were found significant for all traits except seed filling per cent. The significance of variance due to parents Vs crosses indicated the presence of heterosis in hybrids for all characters except days to $50 \%$ flowering and harvest index. The perusal of gca effects of 12 parents (4 CMS lines and 8 testers) for 11 traits that the NDCMS-1B was a good general combiner for oil content exhibiting significant gca effects. CMS-339/89/1Bwas good general combiner for biological yield in positive direction and for plant height in negative direction exhibiting significant gca effects. CMS-SS-502B was good combiner for head diameter, 100 seed weight, volume weight, seed yield per plant and seed filling percentage exhibiting significant gca effects. Among testers LSF-71-1-9 was good combiner for harvest index, seed yield per plant, and seed filling percentage exhibiting significant gca effects. The hybrid DSFH-4 was having significant sca effect for seed yield per plant, biological yield, harvest index, and seed filling percentage.

\section{Introduction}

Sunflower is one of the important oilseed crop in India. Cultivation of sunflower in India started in 1972 with the introduction of Russian varieties, Peredovick (EC 68414) and Armavirskii (EC 68415). The introduction of the crop in India has helped a great deal in increasing oilseed production and the area under its cultivation is increasing due to its day neutrality, wide adaptability, short duration, high yield potential, remunerative market price and good quality oil.
Although nearly every part of the sunflower has been used by human in one or other way, it is the oil that is most valuable. Sunflower oil, however, is nutritionally better and more stable than soybean oil and hence is better for human consumption. Sunflower oil is considered as premium oil because of its light colour, flavour, high smoke point and good nutritional quality. The oil concentration in whole seed varies from 30 to 45 per cent depending on the genotype and growing conditions. The high percentage of polyunsaturated fatty acids in the oil renders it 
is a source of essential fatty acids in certain diets. Sunflower oil has high and moderate levels of linoleic acid (40-67\%) and oleic acid (22-50\%) respectively. It contains less than $15 \%$ saturated fatty acids viz., palmitic and myristic acids. High proportion of the essential fatty acid is considered to reduce blood cholesterol and therefore reduce risk of coronary heart disease, hence sunflower has a special significance. High oleic sunflower oil has higher degree of oxidative stability than oil low in oleic acid (Fuller et al., 1967), which is desirable for frying purposes, refining and storage. The higher level of compounds present in sunflower seed viz., tocopherols (vit E), choline, betaine and phenolic acid makes "the sunflower a power house of health benefits. In India sunflower is cultivated on an area of 8.20 lakh hectares with a production of 5.80 lakh tones (Annual report 2014). The average yield of $705 \mathrm{~kg} / \mathrm{ha}$ is one of the lowest in world. In Bihar sunflower is cultivated on an area of 17.9 thousand hectare with production of 25.4 thousand tones with a productivity of 1416 $\mathrm{kg} / \mathrm{ha}$ (anonymous; 2014).

With the increase in demand for edible oils, there is a need to develop new sunflower hybrids suited to different agro-climatic zones of India with improved seed yield and oil content. Therefore, it is essential to understand the genetic architecture of sunflower, which provides useful guideline to determine the source population and from which it is possible to derive appropriate genotypes with desired characters. The selection of parents is one of the important aspects in developing a potential hybrid which is practiced after testing of parents for their combining ability effects. It is also useful in understanding the type of gene action controlling various traits to develop suitable breeding strategy. Several biometrical techniques have been developed to generate information on gene action and mode of inheritance of various characters, among which linextester analysis (Kempthorne, 1957) has been widely used for genetic analysis in large number of crop plants. It is very efficient technique for evaluating large number of inbred lines for their combining ability; besides, this technique also provides information on general (gca) and specific (sca) combining ability, which are useful to study gene action controlling various characters to adopt appropriate breeding strategy.

\section{Materials and Methods}

The experimental material consists of four female lines and eight restorers were crossed in Line X Tester model to produce 32 hybrids. The resulting 32 hybrids, 12 parents and two checks were studied in randomized block design with three replications during Rabi/Spring 2014-2015 at oilseeds Research Farm, Dholi. Each plot consisted of three rows of 3.0 meter length with a spacing of $60 \mathrm{~cm}$ between rows and $30 \mathrm{~cm}$ within row. The recommended dose of fertilizer was applied 60:90:60 kg NPK/ha. The recommended cultural practices including plant protection measures were followed. The morphological observations on 11 quantitative characters were recorded by selecting randomly 5 competitive plants in each plot.

During the rabi season of 2014 , all the $\mathrm{F}_{1}$ 's were obtained by crossing the cytoplasmic male sterile lines with restorer lines. The cytoplasmic male sterile lines and the restorer lines were sown in the field to affect crossing. At the time of flowering, all the heads of lines and testers were covered with muslin cloth bags to prevent open pollination. The pollen of the eight restorer lines was collected separately in petri dishes with the help of camel hairbrush during morning hours $(9.00$ to $11.00 \mathrm{am})$ and pollinated to each of the cytoplasmic male sterile lines separately and cloth bag was replaced after pollination. Pollination was done till all the florets in the 
capitulum sowed sign of drying. The heads of all the resultant 32 hybrids were collected, dried and threshed. The well filled seeds from each cross were separated out for hybrid evaluation. The complete set of experimental material comprised of 46 genotypes i.e. 4 CMS lines, 8 restorer lines, 32 hybrids and 2 standard checks were sown in RBD with two replications during Rabi/Spring 2014-2015. Each genotype was sown in three rows, each of 3 meter length. Spacing $60 \times 30$ was followed for sowing. All recommended package of practices were followed to raise the healthy crop.

\section{Harvest index (\%)}

The ratio of seed yield to biological yield is Harvest Index and expressed in percentage

Harvest Index $(\%)=\frac{\text { Seed yeild }}{\text { Biological yeild }} \times 100$

\section{Seed filling (\%)}

It is the per cent ratio of number of filled seeds to the total seeds of each selected plants in each treatment were counted and then and seed filling percentage was calculated.

Per cent of seed set $=$

$\frac{\text { Number of well filled seeds in head }}{\text { Number of total seeds (filled }+ \text { unfilled) }}$

\section{Oil content $(\%)$}

Random bulk samples of filled seeds were drawn from selected plant produced weighing 15 grams. The percentage was measured using NMR facilities available at DOR, Hyderabad.

\section{Results and Discussion}

The analysis of variances (Table 1) showed highly significant differences among the genotypes for all the characters indicating the existence of sufficient variability and diversity among the parents. The magnitude of mean sum of square particularly for plant height, biological yield per plant (g), volume weight, and seed filling percentage were high and it was less for 100 seed weight, oil content (\%) and days to $50 \%$ flowering.

Combining ability analysis helps the breeder in selecting desirable parents for exploitation in breeding programme. Number of studies have been reported for evaluating the CMS lines and restores. Use of general combining ability i.e. gca effects of the parents is the important criteria for selection. Generally the parents with high per se performance may not transmit their superiority in their progenies. Hence it is essential to assess the compatibility of parents to express their own high performance to the hybrids involving them.

The analysis of variance for parents and crosses for 11 characters were presented in (Table 2). The mean sum of squares for parents and crosses, were found significant for all traits except seed filling per cent. The significance of variance due to parents Vs crosses indicated the presence of heterosis in hybrids for all characters except days to $50 \%$ flowering and harvest index. Similar results were also reported by Halaswamy et al., (2004) and Ravi-Rana et al., (2004).

Mean sum of square due to lines and testers in analysis of variance for combining ability (Table 3) indicated that the variance for combining ability was significant among all traits except 100 seed weight and seed filling per cent in case of lines and except seed yield per plant in case of testers indicating the preponderance of additive gene action in the expression of these traits.

Variances due to lines, testers, lines $\mathrm{x}$ tester interaction (Table 3) indicated that there was significant variation for combining ability for 
all the traits among crosses and line $\mathrm{x}$ tester effect except oil content suggesting the presence of non- additive gene action in expression of these traits.

The gca variance of males was higher than that of females for all characters except 100 seed weight and seed filling per cent indicating the existence of wide genetic diversity and preponderance of additive variance through males and females for the respective trait. The variance component due to specific combining ability (sca) was greater in magnitude than that of general combining ability (gca) (Table 3) for all the characters except oil content indicating the predominance of non-additive type of gene action in expression of these traits which is in agreement with the Dua and Yadava (1983) Kadkol et al., (1984) Kumar et al., (1998) Ashok et al.,(2000), Radhika et al., (2001) and Ortis et al., (2005).

Per cent contribution of male lines were highest for traits harvest index (55.92), biological yield (54.45) and head diameter (52.18) while the contribution of interaction was maximum for traits days to $50 \%$ flowering (70.32), days to maturity (69.46), 100 seed weight (63.69), seed yield per plant (62.55), plant height (58.59) and seed filling per cent (51.34).

\section{Estimates of general combining ability effects}

General and specific combining ability effects helps to pin point the good parent and hybrids, respectively. The perusal of gca effects of 12 parents (4 CMS lines and 8 testers) for 11 traits indicated (Table 4 and 5) that the NDCMS-1B was a good general combiner for oil content exhibiting significant gca effects. CMS-339/89/1Bwas good general combiner for biological yield in positive direction and for plant height in negative direction exhibiting significant gca effects. CMS-17B was good combiner for day to $50 \%$ flowering and plant height in negative direction and for 100 seed weight, volume weight and seed filling percentage in positive direction exhibiting significant gca effects. CMS-SS502B was good combiner for head diameter, 100 seed weight, volume weight, seed yield per plant and seed filling percentage exhibiting significant gca effects.

Among testers DRSF-108-13 was good combiner for biological yield and head diameter exhibiting significant gca effects. LSF-71-1-9 was good combiner for harvest index, seed yield per plant, and seed filling percentage exhibiting significant gca effects. CSFI-52315 was good combiner for early flowering, early maturity and volume weight exhibiting significant gca effects. CSFI-5313 was good combiner for dwarf plant height, harvest index, 100 seed weight and seed yield per plant exhibiting significant gca effects. GAUSUF-15 was good combiner for early flowering, dwarf plant height, early maturity along with 100 seed weight exhibiting significant gca effects. SS-502-1-1 was good combiner for biological yield, head diameter, volume weight, seed yield per plant and oil content exhibiting significant gca effects.

TNUSUF-7B-2-1 was good combiner for head diameter, volume weight and oil content exhibiting significant gca effects. Genepool-27-1 was good combiner for biological yield and seed filling percentage exhibiting significant gca effects.

All these testers can be exploited for development of better hybrids and also in conventional breeding programme. Such type of good general combiner for economic trait was also reported by Kadkol et al., (1984), Kshirsager et al., (1993), Ortis et al., (2005), and Parmeshwarappa et al., (2008) (Table 6 and 7). 
Table.1 Analysis of variance for eleven characters in sunflower

\begin{tabular}{|c|c|c|c|c|}
\hline \multirow{2}{*}{$\begin{array}{l}\text { Sl. } \\
\text { No. }\end{array}$} & \multirow[t]{2}{*}{ Character } & \multicolumn{3}{|c|}{ Source of variation } \\
\hline & & $\begin{array}{l}\text { Replication } \\
(\mathbf{d f}=2)\end{array}$ & $\begin{array}{l}\text { Treatment } \\
(\mathbf{d f}=45)\end{array}$ & $\begin{array}{c}\begin{array}{c}\text { Error } \\
(d f=90)\end{array}\end{array}$ \\
\hline 1 & Days to $50 \%$ flowering & 4.87 & $1351.72 * *$ & 223.13 \\
\hline 2 & Plant height $(\mathrm{cm})$ & 542.61 & $110585.12 * *$ & 9495.97 \\
\hline 3 & Days to maturity & 0.69 & $3035.27 * *$ & 292.64 \\
\hline 4 & Biological yield per plant (g.) & 89.23 & $157223.08 * *$ & 5667.61 \\
\hline 5 & Head diameter(cm) & 14.88 & $2963.58 * *$ & 249.36 \\
\hline 6 & Harvest index $(\%)$ & 140.64 & $18601.20 * *$ & 2287.10 \\
\hline 7 & 100 seed weight(g.) & 0.58 & $171.77 * *$ & 17.67 \\
\hline 8 & Volume weight(g./l) & 6.31 & $29082.85 * *$ & 415.72 \\
\hline 9 & Seed yield per plant(g.) & 7.5 & $8032.47 * *$ & 1351.84 \\
\hline 10 & Seed filling percent $(\%)$ & 15.95 & $67167.11 * *$ & 1569.12 \\
\hline 11 & Oil content $(\%)$ & 1.86 & $1036.34 * *$ & 249.89 \\
\hline
\end{tabular}

** indicate significance at $1 \%$ 
Table.2 Analysis of variance for parents and crosses of eleven characters in sunflower

\begin{tabular}{|c|c|c|c|c|c|c|c|c|c|}
\hline \multirow[b]{2}{*}{ Character } & \multicolumn{9}{|c|}{ Source of variation } \\
\hline & $\begin{array}{c}\text { Replication } \\
(\mathrm{df}=2)\end{array}$ & $\begin{array}{c}\text { Treatment } \\
(\mathrm{df}=43)\end{array}$ & $\begin{array}{l}\text { Parent } \\
(\mathrm{df}=11)\end{array}$ & $\begin{array}{l}\text { CMS line } \\
(\mathrm{df}=3)\end{array}$ & $\begin{array}{l}\text { Tester } \\
(\mathrm{df}=7)\end{array}$ & $\begin{array}{c}\text { Line v/s Tester } \\
(\mathrm{df}=1)\end{array}$ & $\begin{array}{c}\text { Parent v/s Cross } \\
(\mathrm{df}=1)\end{array}$ & $\begin{array}{c}\text { Cross } \\
(\mathrm{df}=31)\end{array}$ & $\begin{array}{c}\text { Error } \\
(\mathrm{df}=86)\end{array}$ \\
\hline Days to $50 \%$ flowering & 4.36 & $30.34 * *$ & $17.52 * *$ & $14.75^{* *}$ & $20.57 * *$ & 4.50 & 9.23 & $35.56 * *$ & 2.36 \\
\hline Plant height $(\mathrm{cm})$ & 85.52 & $1330.33 * *$ & $1229.23 * *$ & $1466.77 * *$ & $1297.47 * *$ & 39.01 & $6030.43 * *$ & $1214.59 * *$ & 77.87 \\
\hline Days to maturity & 0.29 & $69.161^{* *}$ & $12.13 * *$ & $12.75^{*}$ & $13.66^{* *}$ & 0.13 & $44.38 * *$ & $90.17 * *$ & 3.2 \\
\hline Biological yield per plant (g.) & 69.82 & $3634.8 * *$ & $901.14 * *$ & $1687.40 * *$ & $639.42 * *$ & $378.12 *$ & $16349.73 * *$ & $4194.58 * *$ & 58.25 \\
\hline Head diameter(cm) & $9.51 *$ & $66.307 * *$ & $68.49 * *$ & $26.88 * *$ & $65.69 * *$ & $212.97 * *$ & $376.62 * *$ & $55.51 * *$ & 2.57 \\
\hline Harvest index $(\%)$ & 62.85 & $432.11 * *$ & $132.11 * *$ & $271.27 * *$ & $78.66^{* *}$ & 88.77 & 11.87 & $552.12 * *$ & 26.31 \\
\hline 100 seed weight(g.) & 0.37 & $3.99 * *$ & $1.51 * *$ & 0.27 & $1.99 * *$ & $1.84 * *$ & $56.79 * *$ & $3.166^{* *}$ & 0.20 \\
\hline Volume weight(g./l) & 2.49 & $650.67 * *$ & $26.82 * *$ & $46.22 * *$ & $15.54 * *$ & $47.53 * *$ & $16133.58 * *$ & $372.59 * *$ & 4.77 \\
\hline Seed yield per plant(g.) & 2.94 & $183.33 * *$ & $54.87 * *$ & $128.30 * *$ & 30.91 & 2.21 & $1578.24 * *$ & $183.92 * *$ & 15.22 \\
\hline Seed filling percent $(\%)$ & 7.87 & $1467.60 * *$ & 34.17 & 21.77 & $39.36^{*}$ & 35.06 & $44249.53 * *$ & $596.17 * *$ & 18.00 \\
\hline Oil content $(\%)$ & 1.35 & $23.98 * *$ & $27.98 * *$ & $15.91 * *$ & $33.69 * *$ & $15.90 *$ & $409.00 * *$ & $10.40 * *$ & 2.78 \\
\hline
\end{tabular}

** and * indicates significant at $1 \%$ and $5 \%$ respectively. 
Table.3 Analysis of variance for combining ability (Line $\times$ Tester) of eleven characters in sunflowers

\begin{tabular}{|c|c|c|c|c|c|c|}
\hline Character & $\begin{array}{l}\text { Replication } \\
(\mathbf{d f}=2)\end{array}$ & $\begin{array}{c}\text { Cross } \\
(\mathbf{d f}=\mathbf{3 1})\end{array}$ & $\begin{array}{l}\text { Line effect } \\
\quad(\mathbf{d f}=3)\end{array}$ & $\begin{array}{l}\text { Tester effect } \\
\quad(\mathrm{df}=7)\end{array}$ & $\begin{array}{c}\text { Line } x \text { tester } \\
\text { effect } \\
(d f=21)\end{array}$ & $\begin{array}{c}\text { Error } \\
(\mathrm{df}=62)\end{array}$ \\
\hline Days to $50 \%$ flowering & 2.34 & $35.56^{* *}$ & 24.12 & 36.41 & $36.92 * *$ & 2.67 \\
\hline Plant height(cm) & 225.93 & $1214.6^{* *}$ & 1732.21 & 1465.53 & 1057.** & 80 \\
\hline Days to maturity & 0.50 & $90.17 * *$ & 7.09 & 118.90 & $92.47 * *$ & 4.06 \\
\hline Biological yield per plant(g.) & 173.24 & $4194.58 * *$ & 543.65 & $10114.3^{* *}$ & $2742.88 * *$ & 56.81 \\
\hline Head diameter(cm) & 5.67 & $55.51 * *$ & 18.24 & $128.30 *$ & $36.58 * *$ & 3.01 \\
\hline Harvest index $(\%)$ & 42.51 & $552.12 * *$ & 51.92 & $1367.20 * *$ & $351.88 * *$ & 28.91 \\
\hline 100 seed weight(g.) & 0.01 & $3.16^{* *}$ & 8.21 & 1.56 & $2.97 * *$ & 0.19 \\
\hline Volume weight(g./l) & 1.08 & $372.59 * *$ & 759.14 & 535.76 & $262.98 * *$ & 5.71 \\
\hline Seed yield per plant(g.) & 4.50 & $183.92 * *$ & $96.79^{*}$ & 263.52 & $169.83 * *$ & 18.71 \\
\hline Seed filling percent $(\%)$ & 17.30 & $596.17 * *$ & $2111.71 * *$ & 379.64 & $451.84 * *$ & 23.15 \\
\hline Oil content $(\%)$ & 1.19 & $10.40 * *$ & $25.91 * *$ & $20.82 * *$ & 4.71 & 3.30 \\
\hline
\end{tabular}

$* *$ and * indicates significant at $1 \%$ and $5 \%$ respectively 
Table.4 Variance for general and specific combining ability

\begin{tabular}{|c|c|c|c|c|c|}
\hline Character & $\delta^{2} \operatorname{gca}(\mathbf{f})$ & $\delta^{2} \operatorname{gca}(\mathbf{m})$ & $\delta^{2}$ gca(ave.) & $\delta^{2}$ sca & $\delta^{2} \mathrm{gca} / \mathrm{sca}$ \\
\hline Days to $50 \%$ flowering & 0.91 & 2.84 & 1.55 & $11.52 * *$ & 0.13 \\
\hline Plant height(cm) & 68.93 & 115.63 & $84.50^{*}$ & $326.37 * *$ & 0.26 \\
\hline Days to maturity & 0.16 & 9.63 & 3.32 & $29.73 * *$ & 0.11 \\
\hline Biological yield per plant (g.) & 20.23 & $838.00 * *$ & $292.82 * *$ & $894.87 * *$ & 0.33 \\
\hline Head diameter (cm) & 0.65 & $10.48^{*}$ & $3.93 * *$ & $11.33 * *$ & 0.35 \\
\hline Harvest index $(\%)$ & 1.07 & $111.74 * *$ & $37.96 * *$ & $108.52 * *$ & 0.35 \\
\hline 100 seed weight (g.) & 0.33 & 0.11 & $0.26 *$ & $0.93 * *$ & 0.28 \\
\hline Volume weight (g./l) & 31.43 & 44.25 & $35.70 * *$ & $86.07 * *$ & 0.41 \\
\hline Seed yield per plant (g.) & 3.39 & 20.69 & 9.16 & $51.54 * *$ & 0.18 \\
\hline Seed filling percent (\%) & $87.24 *$ & 30.14 & $68.20 * *$ & $144.61 * *$ & 0.47 \\
\hline Oil content (\%) & $0.96 * *$ & $1.50 * *$ & $1.14 * *$ & 0.64 & 1.77 \\
\hline
\end{tabular}

$* *$ and $*$ indicates significant at $1 \%$ and $5 \%$ respectively 
Table.5 Percent contribution of line, tester and their interactions $(\mathrm{L} \times \mathrm{T})$ to hybrid sum of square in sunflower

\begin{tabular}{|c|c|c|c|}
\hline Character & Line & Tester & Line $x$ Tester \\
\hline Days to $50 \%$ flowering & 6.56 & 23.11 & 70.32 \\
\hline Plant height(cm) & 13.80 & 27.24 & 58.95 \\
\hline Days to maturity & 0.76 & 29.77 & 69.46 \\
\hline Biological yield per plant(g.) & 1.25 & 54.45 & 44.29 \\
\hline Head diameter $(\mathrm{cm})$ & 3.20 & 52.18 & 44.63 \\
\hline Harvest index $(\%)$ & 0.91 & 55.92 & 43.17 \\
\hline 100 seed weight (g.) & 25.12 & 11.19 & 63.69 \\
\hline Volume weight (g./l) & 19.71 & 32.46 & 47.81 \\
\hline Seed yield per plant (g.) & 5.09 & 32.35 & 62.55 \\
\hline Seed filling percent (\%) & 34.27 & 1.37 & 51.34 \\
\hline Oil content $(\%)$ & 24.11 & 45.20 & 30.68 \\
\hline
\end{tabular}


Table.6 Estimates of General combining ability (gca) of lines for eleven characters in sunflower

\begin{tabular}{|c|c|c|c|c|c|}
\hline Character & NDCMS-1B & CMS-339/89/1B & CMS-17B & CMS-SS-502B & CD at $5 \%$ \\
\hline Days to $50 \%$ flowering & 0.24 & $1.07 * *$ & $-1.34 * *$ & 0.03 & 0.63 \\
\hline Plant height(cm) & 0.45 & $-3.60 *$ & $-8.35 * *$ & $11.55 * *$ & 3.60 \\
\hline Days to maturity & -0.72 & 0.53 & 0.28 & -0.09 & 0.74 \\
\hline Biological yield per plant (g.) & -2.36 & $6.64 * *$ & $-4.25 * *$ & 0.01 & 3.11 \\
\hline Head diameter $(\mathrm{cm})$ & -0.15 & -0.47 & -0.65 & $1.27 * *$ & 0.65 \\
\hline Harvest index $(\%)$ & -0.56 & -0.39 & -1.56 & 1.77 & 2.10 \\
\hline 100 seed weight (g.) & $-0.54 * *$ & $-0.39 * *$ & $0.74 * *$ & $0.19 *$ & 0.18 \\
\hline Volume weight (g./l) & $-6.45^{* *}$ & $-2.45^{* *}$ & $6.42 * *$ & $2.48 * *$ & 0.89 \\
\hline Seed yield per plant (g.) & 0.68 & -0.16 & $-2.66^{* *}$ & $2.14 * *$ & 1.60 \\
\hline Seed filling percent $(\%)$ & $-11.34 * *$ & $-3.43 * *$ & $4.68 * *$ & $10.09 * *$ & 1.70 \\
\hline Oil content $(\%)$ & $1.32 * *$ & -0.45 & $-1.18 * *$ & 0.25 & 0.68 \\
\hline
\end{tabular}

** and *indicate significance at $1 \%$ and $5 \%$ respectively 
Table.7 Estimation of general combining ability (gca) of testers for eleven characters in sunflower

\begin{tabular}{|c|c|c|c|c|c|c|c|c|c|}
\hline Character & $\begin{array}{c}\text { DRSF-108- } \\
13 \\
\text { (R) }\end{array}$ & $\begin{array}{c}\text { LSF-71-1- } \\
9 \\
(\mathbf{R})\end{array}$ & $\begin{array}{l}\text { CSFI- } \\
52315 \\
(\mathbf{R})\end{array}$ & $\begin{array}{c}\text { CSFI- } \\
5313 \\
(\mathbf{R})\end{array}$ & $\begin{array}{c}\text { GAUSUF- } \\
15 \\
(\mathbf{R})\end{array}$ & $\begin{array}{c}\text { SS-502-1- } \\
1 \\
(\mathbf{R})\end{array}$ & $\begin{array}{c}\text { TNUSUF-7B-2-1 } \\
\text { (R) }\end{array}$ & $\begin{array}{c}\text { Genepool-2-7- } \\
1 \\
(\mathbf{R})\end{array}$ & $\begin{array}{c}\text { CD at } \\
5 \%\end{array}$ \\
\hline Days to $50 \%$ flowering & 0.65 & $1.41^{* *}$ & $-2.68 * *$ & $1.66 * *$ & $-2.59 * *$ & $1.32 * *$ & 0.57 & -0.34 & 0.89 \\
\hline Plant height(cm) & 1.85 & $6.26^{*}$ & -4.26 & $-15.45 * *$ & $-10.13 * *$ & -0.85 & 1.38 & $21.21 *$ & 5.00 \\
\hline Days to maturity & -0.30 & $6.28 * *$ & $-2.89 * *$ & 1.03 & $-3.97 * *$ & $1.28 *$ & 0.28 & -1.72 & 1.00 \\
\hline Biological yield per plant (g) & $12.59 * *$ & $-13.99 * *$ & $-15.32 * *$ & $-33.24 * *$ & $-21.07 *$ & $59.67 * *$ & -2.57 & $13.93 * *$ & 4.40 \\
\hline Head diameter (cm) & $2.27 * *$ & $-1.86 * *$ & $-2.29 * *$ & $-3.04 * *$ & $-3.57 * *$ & $5.47 * *$ & $3.01 * *$ & 0.02 & 0.93 \\
\hline Harvest index(\%) & $-9.65 * *$ & $9.10 * *$ & 0.41 & $20.58 * *$ & 2.12 & $-8.95 * *$ & $-3.55^{*}$ & $-10.06^{* *}$ & 2.90 \\
\hline 100 seed weight (g.) & $-0.32 *$ & $-0.57 * *$ & $-0.26^{*}$ & $0.52 * *$ & $0.27 *$ & 0.17 & -0.02 & 0.21 & 0.26 \\
\hline Volume weight (g./l) & $-6.13^{* *}$ & 3.34 & $2.89 * *$ & $-4.82 * *$ & $-5.69 *$ & $13.35^{* *}$ & $1.48 *$ & $-4.42 * *$ & 1.26 \\
\hline Seed yield per plant (g.) & $-2.62 *$ & $7.72 * *$ & $-3.28 * *$ & $3.97 * *$ & $-5.53 * *$ & $4.39 * *$ & -2.03 & $-2.62 *$ & 2.25 \\
\hline Seed filling percent $(\%)$ & $-3.88 * *$ & $6.39 * *$ & $-8.13 * *$ & -1.46 & 1.50 & $-3.56 * *$ & 0.07 & $9.08 * *$ & 2.45 \\
\hline Oil content $(\%)$ & 0.49 & -0.55 & 0.56 & $-1.51 * *$ & $-1.18^{*}$ & $0.99 *$ & $2.32 * *$ & $-1.12 *$ & 0.96 \\
\hline
\end{tabular}

$* *$ and $*$ indicate significance at $1 \%$ and $5 \%$ respectively 
Table.8 Estimates of specific combining ability (sca) effect for eleven characters in sunflower

\begin{tabular}{|c|c|c|c|c|c|c|c|c|c|c|c|c|}
\hline $\begin{array}{l}\text { Sl. } \\
\text { No. }\end{array}$ & $\begin{array}{l}\text { Character } \\
\text { Cross }\end{array}$ & $\begin{array}{c}\text { Days to } \\
\mathbf{5 0 \%} \\
\text { Flowering }\end{array}$ & $\begin{array}{c}\text { Plant } \\
\text { Height } \\
\text { (cm) }\end{array}$ & $\begin{array}{l}\text { Days to } \\
\text { Maturity }\end{array}$ & $\begin{array}{l}\text { Biological } \\
\text { yield per } \\
\text { plant (g.) }\end{array}$ & $\begin{array}{c}\text { Head } \\
\text { Diameter } \\
(\mathbf{c m})\end{array}$ & $\begin{array}{c}\text { harvest } \\
\text { Index } \\
(\%)\end{array}$ & $\begin{array}{c}100 \text { Seed } \\
\text { Weight } \\
\text { (g.) }\end{array}$ & $\begin{array}{c}\text { Volume } \\
\text { Weight } \\
\text { (g./l.) }\end{array}$ & $\begin{array}{c}\text { Seed } \\
\text { yield Per } \\
\text { Plant (g.) }\end{array}$ & $\begin{array}{c}\text { Seed } \\
\text { Filling } \\
(\%)\end{array}$ & $\begin{array}{c}\text { Oil } \\
\text { Content } \\
(\%)\end{array}$ \\
\hline 1 & DSFH-1 & $-5.74 * *$ & -9.29 & $-2.95 * *$ & $-15.05 * *$ & -1.35 & 1.13 & -0.08 & $-9.26 * *$ & $-5.09^{*}$ & 1.05 & 0.10 \\
\hline 2 & DSFH-2 & $-6.49 * *$ & $-16.25 * *$ & $-11.53 * *$ & $-15.47 * *$ & -0.32 & -1.34 & 0.36 & $-5.77 * *$ & $-12.43 * *$ & -4.89 & 0.17 \\
\hline 3 & DSFH-3 & 0.59 & 0.28 & 0.64 & -8.14 & -0.61 & 0.30 & $-0.54 *$ & 0.25 & -4.09 & $-10.70 * *$ & 0.29 \\
\hline 4 & DSFH-4 & $6.26 * *$ & 5.47 & $8.72 * *$ & $12.78^{* *}$ & -1.57 & $9.39 * *$ & 0.25 & $10.23 * *$ & $15.32 * *$ & $15.63 * *$ & 0.43 \\
\hline 5 & DSFH-5 & $2.51 * *$ & -2.85 & 0.72 & 1.62 & -0.08 & $-8.82 * *$ & -0.37 & -1.03 & $-6.51 * *$ & -1.66 & -0.23 \\
\hline 6 & DSFH-6 & $3.26 * *$ & $10.20^{*}$ & $4.47 * *$ & $34.86 * *$ & $5.13 * *$ & $-6.19 *$ & -0.47 & 1.40 & $9.24 * *$ & $-12.61 * *$ & 0.60 \\
\hline 7 & DSFH-7 & 1.34 & 9.30 & 1.47 & -4.22 & $3.41 * *$ & 4.75 & $0.52 *$ & -1.53 & 2.66 & $15.10 * *$ & $-3.07 * *$ \\
\hline 8 & DSFH-8 & -1.74 & 3.14 & -1.53 & -6.39 & $-4.60 * *$ & 0.77 & 0.32 & $5.72 * *$ & 0.91 & -1.91 & 1.71 \\
\hline 9 & DSFH-9 & $2.43 * *$ & -6.42 & 1.80 & $-10.05^{*}$ & 0.64 & 0.93 & $-0.74 * *$ & $8.60 * *$ & -0.93 & $8.13 * *$ & -0.14 \\
\hline 10 & DSFH-10 & 0.68 & $25.37 * *$ & $-2.78 *$ & $12.86^{* *}$ & 1.21 & 0.87 & 0.31 & $-12.57 * *$ & $8.74 * *$ & 3.45 & -0.44 \\
\hline 11 & DSFH-11 & -1.57 & -8.53 & 0.39 & $-11.80 * *$ & $2.09 *$ & $10.70 * *$ & 0.00 & $7.02 * *$ & $7.07 * *$ & 0.43 & 0.49 \\
\hline 12 & DSFH-12 & 0.43 & $-22.56^{* *}$ & 1.47 & $-18.22 * *$ & -1.00 & $7.80 *$ & -0.06 & $-7.37 * *$ & -1.84 & 1.87 & -1.14 \\
\hline 13 & DSFH-13 & $-3.32 * *$ & $-10.77^{*}$ & -1.53 & $-12.05 * *$ & $-2.85 * *$ & 1.32 & 0.03 & -1.40 & -0.34 & 4.12 & 1.20 \\
\hline 14 & DSFH-14 & -0.24 & $18.96 * *$ & -0.78 & $41.53 * *$ & $-2.96 * *$ & $-15.53 * *$ & -0.13 & -0.27 & $-8.26 * *$ & $14.81 * *$ & 0.20 \\
\hline 15 & DSFH-15 & $2.18 *$ & 3.72 & 1.22 & 1.11 & -0.51 & -3.50 & $0.56^{*}$ & $3.10^{*}$ & -1.18 & $-27.65^{* *}$ & -0.30 \\
\hline 16 & DSFH-16 & -0.57 & 0.22 & 0.22 & -3.39 & $3.39 * *$ & -2.59 & 0.03 & $2.89 *$ & -3.26 & $-5.16^{*}$ & 0.14 \\
\hline
\end{tabular}


Table.8 Contd...

\begin{tabular}{|c|c|c|c|c|c|c|c|c|c|c|c|c|}
\hline $\begin{array}{l}\text { Sl. } \\
\text { No. }\end{array}$ & $\begin{array}{l}\text { Character } \\
\text { Cross }\end{array}$ & $\begin{array}{c}\text { Days to } \\
50 \% \\
\text { Flowering }\end{array}$ & $\begin{array}{c}\text { Plant } \\
\text { Height } \\
\text { (cm) }\end{array}$ & $\begin{array}{l}\text { Days to } \\
\text { Maturity }\end{array}$ & $\begin{array}{c}\text { Biological } \\
\text { Yield } \\
\text { (g./Plant) }\end{array}$ & $\begin{array}{c}\text { Head } \\
\text { Diameter } \\
(\mathrm{cm})\end{array}$ & $\begin{array}{c}\text { harvest } \\
\text { Index } \\
(\%)\end{array}$ & $\begin{array}{l}100 \text { Seed } \\
\text { Weight } \\
\text { (g) }\end{array}$ & $\begin{array}{c}\text { Volume } \\
\text { Weight } \\
\text { (g./l.) }\end{array}$ & $\begin{array}{c}\text { Seed } \\
\text { yield per } \\
\text { plant (g.) }\end{array}$ & $\begin{array}{c}\text { Seed } \\
\text { Filling } \\
(\%)\end{array}$ & $\begin{array}{c}\text { Oil } \\
\text { Content } \\
(\%)\end{array}$ \\
\hline 17 & DSFH-17 & $4.84 * *$ & $14.30 * *$ & $3.72 * *$ & $19.20 * *$ & 1.81 & 3.16 & $-1.66 * *$ & $7.34 * *$ & $10.57 * *$ & $-10.97 * *$ & -0.13 \\
\hline 18 & DSFH-18 & $3.09 * *$ & 5.55 & $15.47 * *$ & 3.45 & 0.08 & -0.33 & $-0.71 * *$ & $13.87 * *$ & 2.24 & $9.42 * *$ & 0.57 \\
\hline 19 & DSFH-19 & -0.82 & 0.74 & -1.03 & $15.45 * *$ & $2.07 *$ & $-10.04 * *$ & $2.02 * *$ & $-10.51 * *$ & -4.09 & $7.73 * *$ & -1.54 \\
\hline 20 & DSFH-20 & $-4.16 * *$ & $25.72 * *$ & $-7.28 * *$ & $16.03 * *$ & 1.67 & $-20.34 * *$ & -0.07 & $-11.13 * *$ & $-8.34 * *$ & $-23.36 * *$ & 1.53 \\
\hline 21 & DSFH-21 & 0.09 & 5.91 & $-2.28 *$ & $-9.47 *$ & 0.19 & $10.85^{* *}$ & $0.85 * *$ & -1.10 & 0.16 & $-6.30 *$ & -0.80 \\
\hline 22 & DSFH-22 & $-3.82 * *$ & $-52.60 * *$ & $-5.53 * *$ & $-100.22 * *$ & $-8.17 * *$ & $29.05 * *$ & $0.61 *$ & $-10.92 * *$ & $-6.43 * *$ & -0.17 & 0.03 \\
\hline 23 & DSFH-23 & -1.07 & 5.44 & $-3.53 * *$ & $26.37 * *$ & 1.27 & $-10.17 * *$ & -0.26 & $10.44 * *$ & 0.66 & $13.41 * *$ & $2.03 *$ \\
\hline 24 & DSFH-24 & $1.84 *$ & -5.06 & 0.47 & $29.20 * *$ & 1.06 & -2.18 & $-0.79 * *$ & 2.03 & $5.24 *$ & $10.24 * *$ & -1.69 \\
\hline 25 & DSFH-25 & -1.53 & 1.40 & $-2.57 *$ & 5.91 & -1.10 & -5.21 & $2.48 * *$ & $-6.68 * *$ & $-4.55^{*}$ & 1.79 & 0.17 \\
\hline 26 & DSFH-26 & $2.72 * *$ & $-14.68 * *$ & -1.16 & -0.84 & -0.97 & 0.80 & 0.03 & $4.47 * *$ & 1.45 & $-7.98 * *$ & -0.30 \\
\hline 27 & DSFH-27 & $1.80^{*}$ & 7.51 & 0.01 & 4.49 & $-3.54 * *$ & -0.97 & $-1.48 * *$ & $3.25^{*}$ & 1.11 & 2.54 & 0.76 \\
\hline 28 & DSFH-28 & $-2.53 * *$ & -8.63 & $-2.91 * *$ & $-10.59 *$ & 0.90 & 3.15 & -0.13 & $8.27 * *$ & $-5.14 *$ & $5.87 *$ & -0.83 \\
\hline 29 & DSFH-29 & 0.72 & 7.71 & $3.09 * *$ & $19.91 * *$ & $2.74 * *$ & -3.35 & -0.51 & $3.53 * *$ & $6.70 * *$ & 3.84 & -0.16 \\
\hline 30 & DSFH-30 & 0.80 & $23.44 * *$ & 1.84 & $23.82 * *$ & $6.00^{* *}$ & $-7.34 *$ & -0.01 & $9.80 * *$ & $5.45^{*}$ & -2.03 & -0.83 \\
\hline 31 & DSFH-31 & $-2.45^{* *}$ & $-18.46 * *$ & 0.84 & $-23.26 * *$ & $-4.18 * *$ & $8.92 * *$ & $-0.82 * *$ & $-12.00 * *$ & -2.14 & -0.85 & 1.34 \\
\hline \multirow[t]{2}{*}{32} & DSFH-32 & 0.47 & 1.70 & 0.84 & $-19.43 * *$ & 0.15 & 4.00 & 0.44 & $-10.64 * *$ & -2.89 & -3.17 & -0.15 \\
\hline & CD at $5 \%$ & 1.77 & 10.18 & 2.09 & 8.81 & 1.85 & 5.92 & 0.52 & 2.52 & 4.50 & 4.90 & 1.92 \\
\hline
\end{tabular}




\section{Estimates of specific combining ability effects}

In sunflower negative sca effects are considered to be desirable for days to $50 \%$ flowering, plant height and days to maturity. Among 32 crosses, seven hybrids for days to $50 \%$ flowering, six hybrids for plant height and nine hybrids for days to maturity exhibited highly significant sca effects in desirable direction. Similarly positive and significant sca effects were observed in eight hybrids for seed yield per plant, eleven hybrids for biological yields, six hybrids for head diameter, six hybrids for harvest index, three hybrids for 100 seed weight, eleven hybrids for volume weight, nine hybrids for seed filling percentage and one hybrid for oil content. The hybrid DSFH-23 was having significant sca effect in desirable direction for maximum number of traits i.e. early maturity, biological yield, volume weight, seed filling percentage and oil content. The hybrid DSFH4 was having significant sca effect for seed yield per plant, biological yield, harvest index, and seed filling percentage while hybrid DSFH-29 was having significant sca effect for seed yield per plant, biological yield, head diameter and volume weight. The hybrid DSFH-30 was also having significant sca effect for seed yield per plant, biological yield, head diameter and volume weight. For all the traits under study the crosses with significant sca effects in the desirable direction involved parents with high $\mathrm{x}$ high or high $\mathrm{x}$ low or low $\mathrm{x}$ low gca effects indicating high performance of these crosses due to additive, dominance and epispastic gene interaction. The ideal crosses combination to be exploited is one whose high magnitude of sca is present in addition to gca in both or at least in one of the parents. Therefore, the hybrid DSFH-30, DSFH-4 and DSFH-6 can be exploited for seed yield per plant through heterosis breeding. These findings are in agreement to those of Parmeswari et al.,
(2004). The results of Dua and Yadva (1983) sugoor, (1996), Goksoy et al., (2000), Vishwanath et al., (2003) and Parmeshwarappa et al., (2008) which revealed that the hybrids with high sca effects were produced when poor general combiners were crossed (Table 8).

In conclusion, the analysis of variance for combining ability revealed that the average performance of hybrids was different from that of parents as evident from significance of parents vs. crosses for most of the traits. The variance component due to specific combining ability was greater in magnitude than that of general combining ability (gca) for all the characters except oil content indicating predominance of non-additive gene action. The per cent contribution of males in the hybrid was higher than female parents for all the characters except 100 seed weight and seed filling per cent.

The gca effect of parents were related to their per se performance. The estimate of gca effect of one female for days to 50 per cent flowering (CMS-17B) and two for plant height (CMS-339/89/1B,CMS-17B) were highly significant in desirable direction, indicating their best general combining ability. The female line CMS-339/89/1B for Biological yield, CMS-SS-502B for seed yield per plant, head diameter, 100 seed weight, volume weight and seed filling percent, CMS-17B again for plant height, 100 seed weight, volume weight and seed filling percent, NDCMS-1B for oil content were the best general combiners. Among the male parents GAUSUF-15 for days to maturity, LSF-71-1-9 for seed yield per plant, Gene pool-2-7-1 for seed filling per cent and TNUSF-7B-2-1 for oil content were identified as the best combiners.

The crosses showing high desirable sca effect have involved either one or both the parents 
of high gca effect and in turn had high per se performance. The sca effect of DSFH-2 (6.49) for early maturity, DSFH-22 (-52.60) for dwarf plant height, DSFH-4 (15.32) and (15.63) for seed yield per plant and seed filling percentage respectively, DSFH18(13.87) for volume weight and DSFH-25 (2.48) for 100 seed weight were significantly higher to checks, which indicated good specific combination. The cross DSFH-4 exhibited high sca effect for seed yield and simultaneously high significant sca effect for biological yield, harvest index, plant height and seed filling percentage. Similarly DSFH30 for seed yield, biological yield, head diameter and volume weight, DSFH-6 for seed yield, biological yield and head diameter. DSFH-10 for seed yield, days to maturity and biological yield. DSFH-18 for seed yield, volume weight and seed filling percentage. DSFH-29 for seed yield, volume weight, biological yield and head diameter. Therefore, DSFH-4 and DSFH-30 were most ideal cross combinations displaying consistent high sca effect with high per se performance for seed yield and important characters.

\section{References}

Anonymous, 2014., annual progress report, Sunflower, 2013-2014. Directorate of oilseeds research, Rajendranagar, Hyderabad.

Ashoka, S., Mohamad Sheriff, N. and Narayanan, S. L., 2000, Combining ability studies in sunflower (Helianthus annuus L.). Crop Res., Hissar 20 (3): 457-462.

Dua, R.P. and Yadav, T.P., 1983. Combining ability in sunflower. Indian J. Genet., 43: 129-136.

Fuller, M., Diamond, J. and Apple, W.T., 1967, High oleic sunflower oil, stability and chemical modification. J.Am.Oil.Chem.Soc., 44: 264-267.

Goksoy, A. T., Turkec, A. and Turan, Z. M.,
2000, Heterosis and combining ability in sunflower (Helianthus annuus L.). Ind. J. Agric. Sci. 70(8): 525-529.

Halaswamy, K.M., Channakrishnaiah, K.M. and Kulkarni, R.s.2004. Combiing ability studies in sunflower. (Helianthus annuus L) crop. Res Hisar. 28 (1/3):103-112.

Kadkol, G.P., Anand, I.J. and Sharma, R. P., 1984, Combining ability and heterosis in sunflower. Ind. J. Genet. and Plant Breeding., 44: 447-451.

Kempthorne, O., 1957, An Introduction of Genet. Stat., The Iowa University Press.

Kshirsagar, A.R. Deshmukh, D.T. and Dudhe, R.S., 1993. Evaluation of early generation inbreds for combining ability in sunflower. $J$ Maharastra Agric. Univ., 18 (3):464-465.

Kumar, A. A., Ganesh, M. and Janila, P., 1998, Combining ability analysis for yield and yield contributing characters in sunflower (Helianthus annuus L.). Ann. Agric. Res., 19 (4):437-440.

Ortis, L., Nestures, G., Frutos, E. and Machado, N., 2005. Combining ability analysis for agronomic traits in sunflower (Helianthus annus). Helia, 28: 125-134.

Parameshwari, C., Muralidharan, V., Subbalaksmi, B. and Manivannan, M., 2004, Genetic analysis of yield and important traits in sunflower (Helianthus annuus L.) hybrids. J. of Oilseeds Res., 21(1): 168-170.

Parmeshwarppa, K.G., Jalandhar, R. and Lingaraju, B. S. 2008, Heterosis and combining ability for seed yield, oil content other agronomic traits involving mutant restorer lines in sunflower (Helianthus annus L.) J. Oilseeds Res., 25(1):8-12

Radhika, P., Jagadeshwar, K. and Khan, K.A., 2001, Heterosis and combining ability through line $\mathrm{x}$ tester analysis in 
sunflower (Helianthus annuus L.). J. Res., Acharya N. G. Ranga Agric. Univ., 29 (2-3): 35-43.

Ravi-Rana, Sheoran, R. K., Rakul Kumar and Gill, H. S., 2004, Combining ability analysis in sunflower (Helianthus annuus L.). Nation J. Plant Improv., 6 (2): 89-93.

Sugoor, R.K., Giriraj, K., Salimath, P.M.and Rao,N.G.H.1996 Genetic potential of induced mutant restorer lines producing high yielding early maturing sunflower hybrids. Indian J. Genetics, 56910:1620

Vishwanath, K.S., 2003, Evaluation of hybrids synthesized from elite CMS lines and restorer for their value in crop improvement in sunflower (Helianthus annuus L.) M.Sc. (Agri.) Thesis, Uni. Agric. Sci. Bangalore (India).

\section{How to cite this article:}

Singh, U.K., Dhiraj Kumar and Rajesh Kumar. 2018. Determining Combining Ability in Sunflower (Helianthus annus L.). Int.J.Curr.Microbiol.App.Sci. 7(05): 2290-2305.

doi: https://doi.org/10.20546/ijcmas.2018.705.266 